D. Ya. Petrina (Inst. Math. Nat. Acad. Sci. Ukraine, Kyiv),

G. L. Caraffini (Univ. Parma, Italy)

\title{
SOLUTIONS OF THE BBGKY HIERARCHY \\ FOR A SYSTEM OF HARD SPHERES \\ WITH INELASTIC COLLISIONS*
}

\section{РОЗВ'ЯЗКИ ІЕРАРХЇ̈ ББГКІ ДЛЯ СИСТЕМИ ТВЕРДИХ КУЛЬ ІЗ НЕПРУ ЖНИМ РОЗСІЯННЯМ}

The problem of existence of solutions of the hierarchy for the sequence of correlation functions is investigated in direct sum of spaces of summable functions. It is proved existence and uniqueness of solutions which are represented through a semigroup of bounded strongly continuous operators.

Infinitesimal generator of the semigroup coincides, on certain everywhere dense set, with the operator in the right-hand side of the hierarchy. For initial data from this set, solutions are strong; for general initial data, they are generalized ones.

Досліджено проблему існування розв'язків ієрархії для послідовності кореляційних функцій при початкових даних з прямої суми просторів інтегровних функцій. Доведено існування та єдиність розв'язів, поданих через півгрупу обмежених сильно неперервних операторів.

Інфінітезимальний оператор півгрупи збігається на певній скрізь щільній множині з оператором, що визначає праву частину ієрархії. Для початкових даних з цієї множини розв'язкиє строгими, для загальних початкових даних - узагальненими.

Introduction. We consider the analogue of the BBGKY hierarchy for systems of hard spheres with inelastic collisions. It is commonly accepted that these systems are proper models of granular flow. We continue the investigation of these systems that have been begun in our paper [1]. We use the same denotation.

In paper [1] the Liouville equation for distribution functions of systems of finite numbers $N$ of hard spheres with inelastic collisions has been investigated. It has been proved that the distribution function is defined as follows:

$$
D_{N}\left(t,(x)_{N}\right)=\left[\frac{\partial X\left(-t,(x)_{N}\right)}{\partial(x)_{N}}\right]^{2} S_{N}\left(-t,(x)_{N}\right) D\left(0,(x)_{N}\right), \quad N \geq 1,
$$

where $D\left(0,(x)_{N}\right)$ is the initial distribution function, $S_{N}\left(-t,(x)_{N}\right)$ is the operator of shift along the trajectory $X\left(-t,(x)_{N}\right)$ of $N$ spheres with initial data $(x)_{N}, \frac{\partial X\left(-t,(x)_{N}\right)}{\partial(x)_{N}}$ is the Jacobian. It has been shown that only distribution functions (1), with squared Jacobian, satisfy the law of conservation of full probability

$$
\int D_{N}\left(t,(x)_{N}\right) d(x)_{N}=\int D_{N}\left(0,(x)_{N}\right) d x_{N}
$$

the Liouville equation

$$
\frac{\partial}{\partial t} D_{N}\left(t,(x)_{N}\right)=-\sum_{i=1}^{N} p_{i} \frac{\partial}{\partial q_{i}} D_{N}\left(t,(x)_{N}\right),
$$

and specific boundary conditions according to which at $q_{i}-q_{j}=a \eta,\left\langle\eta,\left(p_{i}-p_{j}\right)\right\rangle>$ $>0,\left(|\eta|=1, a\right.$ - diameter of hard spheres) momenta $p_{i}, p_{j}$ should be replaced by the following ones:

$$
p_{i}^{*}=p_{i}+\frac{\varepsilon}{1-2 \varepsilon} \eta\left\langle\eta,\left(p_{i}-p_{j}\right)\right\rangle, \quad p_{j}^{*}=p_{j}-\frac{\varepsilon}{1-2 \varepsilon} \eta\left\langle\eta,\left(p_{i}-p_{j}\right)\right\rangle
$$

* This paper has been completed during stay of D. Ya. Petrina in November 2004 in Dipartimento di Matematica, Università di Parma. 
and

$$
\begin{gathered}
D_{N}\left(-t, x_{1}, \ldots, q_{i}, p_{i}, \ldots, q_{i}-a \eta, p_{j}, \ldots, x_{N}\right)= \\
=\frac{1}{(1-2 \varepsilon)^{2}} D_{N}\left(-t, x_{1}, \ldots, q_{i}, p_{i}^{*}, \ldots, q_{i}-a \eta, p_{j}^{*}, \ldots, x_{N}\right) .
\end{gathered}
$$

The parameter $\varepsilon$, characterizes inelasticity, $\frac{1}{2}<\varepsilon<1$. The corresponding sequence of correlation functions satisfies the following analogue of the BBGKY hierarchy

$$
\begin{aligned}
\frac{\partial}{\partial t} \rho_{s}\left(t,(x)_{N}\right) & =-\sum_{i=1}^{s} p_{i} \frac{\partial}{\partial q_{i}} \rho_{s}\left(t,(x)_{s}\right)+a^{2} \sum_{i=1}^{s} \int d p_{s+1} \int_{S_{2}^{+}} d \eta\left\langle\eta,\left(p_{i}-p_{s+1}\right)\right\rangle \times \\
\times & {\left[\frac{1}{(1-2 \varepsilon)^{2}} \rho_{s+1}\left(t, x_{1}, \ldots, q_{i}, p_{i}^{*}, \ldots, x_{s}, q_{i}-a \eta, p_{s+1}^{*}\right)-\right.} \\
& \left.-\rho_{s+1}\left(t, x_{1}, \ldots, q_{i}, p_{i}, \ldots, x_{s}, q_{i}+a \eta, p_{s+1}\right)\right], \quad s \geq 1
\end{aligned}
$$

with the same boundary condition in the first term on the right-hand side of (6) as those (4), (5) for $D_{N}\left(t,(x)_{N}\right)$ and with the initial conditions

$$
\left.\rho_{s}\left(t,(x)_{s}\right)\right|_{t=0}=\rho_{s}\left(0,(x)_{s}\right), \quad s \geq 1 .
$$

In given paper we consider hierarchy (6) with initial data (7), i.e., the Cauchy problem for the hierarchy (6) in the Banach space $L_{1}$ of sequences of integrable symmetric functions

$$
f=\left(f_{1}\left(x_{1}\right), \ldots, f_{s}\left((x)_{s}\right), \ldots\right)
$$

equal to zero on forbidden configurations where $\left|q_{i}-q_{j}\right|<a$ at least for one pair $(i, j) \subset$ $\subset(1, \ldots, s)$, with norm

$$
\|f\|=\sum_{s=1}^{\infty}\left\|f_{s}\right\|, \quad\left\|f_{s}\right\|=\int\left|f_{s}\left((x)_{s}\right)\right| d(x)_{s} .
$$

The corresponding group of bounded and strongly continuous in $L_{1}$ evolution operators $U(t), t \geq 0$, has been constructed

$$
U(t)=e^{\int d x} J(-t) S(-t) e^{-\int d x},
$$

where

$$
\left(\int d x f\right)_{s}\left((x)_{s}\right)=\int d x_{s+1} f_{s+1}\left(x_{1}, \ldots, x_{s}, x_{s+1}\right)
$$

is a bounded operator in $L_{1},\left\|\int d x\right\| \leq 1, J(-t)$ is direct sum of the operators of multiplication by squared Jacobian

$$
(J(-t) f)_{s}\left((x)_{s}\right)=\left[\frac{\partial X\left(-t,(x)_{s}\right)}{\partial(x)_{s}}\right]^{2} f_{s}\left((x)_{s}\right)
$$

and $S(-t)$ is direct sum of the operators $S_{s}\left(-t,(x)_{s}\right)$ of shift along the trajectory $X\left(-t,(x)_{s}\right)$

$$
(S(-t) f)_{s}\left((x)_{s}\right)=\left(S_{s}\left(-t,(x)_{s}\right) f_{s}\right)\left((x)_{s}\right)=f_{s}\left(X\left(-t,(x)_{s}\right)\right) .
$$


It is proved that group $U(t)$ is strongly differentiable on everywhere dense set $L_{1}^{0} \subset$ $\subset L_{1}$ consisting of finite sequences $f \in L_{1}$ of differentiable functions equal to zero in some neighborhood of forbidden configurations and with compact support. The infinitesimal generator $B$ of the group $U(t)$ coincides on $L_{1}^{0}$ with the operator in the right-hand side of the hierarchy (6).

Denoting by $\rho(t)$ the sequence of correlation functions $\left(\rho_{1}\left(t,(x)_{1}\right), \ldots, \rho_{s}(t\right.$, $\left.\left.\left.(x)_{s}\right), \ldots\right)\right)$, we consider the hierarchy (6) as an abstract evolution equation in $L_{1}$ for the sequence $\rho(t)$

$$
\frac{d \rho(t)}{d t}=B \rho(t),\left.\quad \rho(t)\right|_{t=0}=\rho(0),
$$

and show that the Cauchy problem (12) for the hierarchy (6) has the unique solution

$$
\rho(t)=U(t) \rho(0), \quad \rho(0) \in L_{1},
$$

that is strong for $\rho(0) \in L_{1}^{0}$ and the generalized one for arbitrary $\rho(0) \in L_{1}$.

1. Solution of hierarchy for correlation functions. 1.1. Formulae that express $\rho(t)$ through $\rho(0)$. As known [1] the correlation functions defined by formulae

$$
\begin{gathered}
\rho_{s}\left(t, x_{1}, \ldots, x_{s}\right)=\frac{1}{\Xi} \sum_{n=0}^{\infty} \frac{1}{n !} \int\left[\frac{\partial X\left(-t, x_{1}, \ldots, x_{s}, x_{s+1}, \ldots, x_{s+n}\right)}{\partial\left(x_{1}, \ldots, x_{s}, x_{s+1}, \ldots, x_{s+n}\right)}\right]^{2} \times \\
\times\left[S_{s+n}\left(-t, x_{1}, \ldots, x_{s}, x_{s+1}, \ldots, x_{s+n}\right) \times\right. \\
\left.\times D_{s+n}\left(0, x_{1}, \ldots, x_{s}, x_{s+1}, \ldots, x_{s+n}\right)\right] d x_{s+1} \ldots d x_{s+n}= \\
=\frac{1}{\Xi} \sum_{n=0}^{\infty} \frac{1}{n !} \int\left[\frac{\partial X\left(-t,(x)_{s+n}\right)}{\partial(x)_{s+n}}\right]^{2} \times \\
\times\left[S_{s+n}\left(-t,(x)_{s+n}\right) D_{s+n}\left(0,(x)_{s+n}\right)\right] d(x)_{s+n}^{s}, \quad s \geq 1 \\
\Xi=\sum_{n=0}^{\infty} \frac{1}{n !} \int\left[\frac{\partial X\left(-t,(x)_{n}\right)}{\partial(x)_{n}}\right]^{2}\left[S_{n}\left(-t,(x)_{n}\right) D_{n}\left(0,(x)_{n}\right)\right] d(x)_{n}= \\
=\sum_{n=0}^{\infty} \frac{1}{n !} \int D_{n}\left(0,(x)_{n}\right) d(x)_{n}
\end{gathered}
$$

are formal solutions of the hierarchy

$$
\begin{gathered}
\frac{\partial \rho_{s}\left(t,(x)_{s}\right)}{\partial t}= \\
=-\sum_{i=1}^{s} p_{i} \frac{\partial}{\partial q_{i}} \rho_{s}\left(t,(x)_{s}\right)+a^{2} \sum_{i=1}^{s} \int d p_{s+1} \int_{S_{+}^{2}} d \eta\left\langle\eta,\left(p_{i}-p_{s+1}\right)\right\rangle \times \\
\times\left[\frac{1}{(1-2 \varepsilon)^{2}} \rho_{s+1}\left(t, q_{1}, p_{1}, \ldots, q_{i}, p_{i}^{*}, \ldots, q_{s}, p_{s}, q_{i}-a \eta, p_{s+1}^{*}\right)-\right.
\end{gathered}
$$

ISSN 1027-3190. Укр. мат. журн., 2006, 58, № 3 


$$
\left.-\rho_{s+1}\left(t, q_{1}, p_{1}, \ldots, q_{i}, p_{i}, \ldots, q_{s}, p_{s}, q_{i}+a \eta, p_{s+1}\right)\right], \quad s \geq 1
$$

with corresponding boundary and initial conditions. Recall that we use the same denotation as in our previous paper [1]

$$
\begin{gathered}
(x)_{s+n}=\left(x_{1}, \ldots, x_{s}, x_{s+1}, \ldots, x_{s+n}\right), \\
d(x)_{s+n}^{s}=d x_{s+1} \ldots d x_{s+n}, \quad|\eta|=1, \quad S_{2}^{+}\left(\eta \mid\left\langle\eta,\left(p_{i}-p_{j}\right)\right\rangle>0\right),
\end{gathered}
$$

$S_{s+n}\left(-t,(x)_{s+n}\right)$ is the operator of shift along the trajectory $X_{s+n}\left(-t,(x)_{s+n}\right)$, $D_{s+n}\left(0,(x)_{s}\right)$ is the initial distribution function, $\frac{\partial X\left(-t,(x)_{s+n}\right)}{\partial(x)_{s+n}}$ is the Jacobian, $\Xi$ is the grand partition function.

Correlation function satisfies the following boundary condition: at $q_{i}-q_{j}=a \eta$, $\left\langle\eta,\left(p_{i}-p_{j}\right)\right\rangle>0$ momenta $p_{i}$ and $p_{j}$ in the first term of the right-hand side of (1.2) should be replaced by

$$
p_{i}^{*}=p_{i}+\frac{\varepsilon}{1-2 \varepsilon} \eta\left\langle\eta_{i},\left(p_{i}-p_{j}\right)\right\rangle, \quad p_{j}^{*}=p_{j}-\frac{\varepsilon}{1-2 \varepsilon} \eta\left\langle\eta_{i},\left(p_{i}-p_{j}\right)\right\rangle
$$

in $-\sum_{i=1}^{s} p_{i} \frac{\partial}{\partial q_{i}}$ and $\rho_{s}\left(t,(x)_{s}\right)$ should be replaced by $\frac{1}{(1-2 \varepsilon)^{2}} \rho_{s}\left(t, x_{1}, \ldots, q_{i}, p_{i}^{*}, \ldots\right.$ $\left.\ldots, q_{i}-a \eta, p_{i}^{*}, \ldots, x_{N}\right)$.

In (1.1) the correlation functions $\rho_{s}\left(t,(x)_{s}\right)$ are expressed by the initial distribution functions $D_{s+n}\left(0,(x)_{s+n}\right), n \geq 0$. We transform formulae (1.1) in such a way that $\rho_{s}\left(t,(x)_{s}\right)$ will be expressed by the initial correlation functions $\rho_{s}\left(0,(x)_{s+n}\right), n \geq$ $\geq 0$. To do it, we use (1.1) with $t=0$, when $S\left(0,(x)_{s+n}\right)=I, I-$ unit operator, $\frac{\partial X\left(0,(x)_{s+n}\right)}{\partial(x)_{s+n}}=1$. One obtains

$$
\rho_{s}\left(0,(x)_{s}\right)=\frac{1}{\Xi} \sum_{n=0}^{\infty} \frac{1}{n !} \int D_{s+n}\left(0,(x)_{s+n}\right) d(x)_{s+n}^{s} .
$$

Denote by $\rho(0), D(0)$ and $f$ the following sequences:

$$
\begin{aligned}
\rho(0) & =\left(\rho_{1}\left(0, x_{1}\right), \ldots, \rho_{s}\left(0,(x)_{s}\right), \ldots\right), \\
D(0) & =\left(D_{1}\left(0, x_{1}\right), \ldots, D_{s}\left(0,(x)_{s}\right), \ldots\right), \\
f & =\left(f_{1}\left(x_{1}\right), \ldots, f_{s}\left((x)_{s}\right), \ldots\right),
\end{aligned}
$$

and by $\int d x$ the following operator:

$$
\left(\int d x f\right)_{s}\left((x)_{s}\right)=\int f_{s+1}\left((x)_{s}, x_{s+1}\right) d x_{s+1} .
$$

Formulae (1.3) can be represented as follows:

$$
\begin{gathered}
\rho(0)=\frac{1}{\Xi} e^{\int d x} D(0), \quad \rho_{s}\left(0,(x)_{s}\right)=\frac{1}{\Xi}\left(e^{\int d x} D(0)\right)_{s}(x)_{s}, \\
D(0)=\Xi e^{-\int d x} \rho(0) .
\end{gathered}
$$


Denote by $J(t)$, as in introduction, the direct sum of operators of multiplication of sequences (1.4) by $\left[\frac{\partial X\left(-t,(x)_{s}\right)}{\partial(x)_{s}}\right]^{2}$

$$
(J(-t) f)_{s}\left((x)_{s}\right)=\left[\frac{\partial X\left(-t,(x)_{s}\right)}{\partial(x)_{s}}\right]^{2} f_{s}\left((x)_{s}\right)
$$

and by $S(-t)$ the direct sum of operator $S_{s}\left(-t,(x)_{s}\right)$

$$
(S(-t) f)_{s}\left((x)_{s}\right)=\left(S_{s}\left(-t,(x)_{s}\right) f_{s}\right)\left(x_{s}\right)=f_{s}\left(X\left(-t,(x)_{s}\right)\right) .
$$

In terms of these operators formulae (1.1) can be represented as follows:

$$
\begin{aligned}
\rho(t) & =\frac{1}{\Xi} e^{\int d x} J(-t) S(-t) D(0), \\
\rho_{s}\left(t,(x)_{s}\right) & =\frac{1}{\Xi}\left(e^{\int d x} J(-t) S(-t) D(0)\right)_{s}(x)_{s} .
\end{aligned}
$$

Finally, expressing $D(0)$ in (1.7) through $\rho(0)$, according to (1.6), we have

$$
\begin{gathered}
\rho(t)=e^{\int d x} J(-t) S(-t) e^{-\int d x} \rho(0)=U(t) \rho(0), \\
U(t)=e^{\int d x} J(-t) S(-t) e^{-\int d x}
\end{gathered}
$$

or, component-wise,

$$
\begin{gathered}
\rho_{s}\left(t,(x)_{s}\right)=\sum_{n=0}^{\infty} \sum_{k=0}^{n} \frac{(-1)^{k}}{(n-k) ! k !} \int\left[\frac{\partial X\left(-t,(x)_{s+n-k}\right)}{\partial(x)_{s+n-k}}\right]^{2} \times \\
\times S_{s+n-k}\left(-t,(x)_{s+n-k}\right) \rho_{s+n}\left(0,(x)_{s+n}\right) d(x)_{s+n}^{s} .
\end{gathered}
$$

$U(t)$ is the evolution operator of hierarchy (1.2). These formulae have been obtained on formal level. In the next subsection the justification of these formulae will be presented.

1.2. Convergence of series (1.8). Suppose that sequence $f(1.4)$ consists of integrable symmetric functions $\int\left|f_{s}\left((x)_{s}\right)\right|=\left\|f_{s}\right\|<\infty$ equal to zero on forbidden configurations and with norm

$$
\|f\|=\sum_{s=1}^{\infty}\left\|f_{s}\right\|<\infty
$$

This means that $f$ belongs to the Banach space $L_{1}$ consisting of sequences of integrable symmetric functions equal to zero on forbidden configurations with norm $\|f\|$ and component-wise linear operations.

In previous paper [1] we proved that

$$
\int\left[\frac{\partial X\left(-t,(x)_{s}\right)}{\partial(x)_{s}}\right]^{2}\left|S\left(-t,(x)_{s}\right) f_{s}\left((x)_{s}\right)\right| d(x)_{s}=\int\left|f_{s}\left((x)_{s}\right)\right| d(x)_{s} .
$$

It means that

$$
\|J(-t) S(-t) f\|=\|f\|
$$

If is obvious that 


$$
\left\|\int d x f\right\| \leq\|f\|
$$

so that

$$
\left\|\int d x\right\| \leq 1, \quad\left\|e^{ \pm \int d x}\right\| \leq e, \quad e^{ \pm \int d x} e^{\mp \int d x}=1 .
$$

Taking into account (1.9) - (1.11) one obtains from (1.8) that

$$
\|U(t) f\|=\left\|e^{\int d x} J(-t) S(-t) e^{-\int d x} f\right\| \leq e^{2}\|f\|
$$

for arbitrary $f \subset L_{1}$ and it means that the operator of evolution $U(t)$ is a bounded one in space $L_{1}$, i.e.,

$$
\|U(t)\| \leq e^{2} .
$$

1.3. Group property of $\boldsymbol{U}(\boldsymbol{t})$. We have proved in [1] that the operator $S(-t)$ has the group property

$$
S\left(t_{1}+t_{2}\right)=S\left(-t_{1}\right) S\left(-t_{2}\right)=S\left(-t_{2}\right) S\left(-t_{1}\right)
$$

for arbitrary $t_{1}>0, t_{2}>0$.

It has also been proved in [1] that the operator $J(-t)$ has the following property:

$$
\begin{gathered}
{\left[\frac{\partial X\left(-t_{1}-t_{2},(x)_{s}\right)}{\partial(x)_{s}}\right]^{2}=\left[\frac{\partial X\left(-t_{1}, X\left(-t_{2},(x)_{s}\right)\right)}{\partial X\left(-t_{2},(x)_{s}\right)} \frac{\partial X\left(-t_{2},(x)_{s}\right)}{\partial(x)_{s}}\right]^{2}=} \\
=\left[\frac{\partial X\left(-t_{2}, X\left(-t_{1},(x)_{s}\right)\right)}{\partial X\left(-t_{1},(x)_{s}\right)} \frac{\partial X\left(-t_{1},(x)_{s}\right)}{\partial(x)_{s}}\right]^{2} .
\end{gathered}
$$

This equality follows from the fact that the Jacobian $\frac{\partial X\left(-t_{1}-t_{2},(x)_{s}\right)}{\partial(x)_{s}}$ is equal to product of the Jacobians that correspond to consecutive time intervals $\left[0, t_{2}\right],\left[t_{2}, t_{2}+t_{1}\right]$ or $\left[0, t_{1}\right],\left[t_{1}, t_{1}+t_{2}\right]$.

Now we show that product of the operators $J(-t)$ and $S(-t)$ satisfies group property

$$
\begin{gathered}
J\left(-t_{1}-t_{2}\right) S\left(-t_{1}-t_{2}\right)=J\left(-t_{1}\right) S\left(-t_{1}\right) J\left(-t_{2}\right) S\left(-t_{2}\right)= \\
=J\left(-t_{2}\right) S\left(-t_{2}\right) J\left(-t_{1}\right) S\left(-t_{1}\right) .
\end{gathered}
$$

Prove (1.15) in $s$-particle subspace. One has

$$
\begin{gathered}
{\left[\frac{\partial X\left(-t_{1},(x)_{s}\right)}{\partial(x)_{s}}\right]^{2} S_{s}\left(-t_{1},(x)_{s}\right)\left\{\left[\frac{\partial X\left(-t_{2},(x)_{s}\right)}{\partial(x)_{s}}\right]^{2} S_{s}\left(-t_{2},(x)_{s}\right)\right\}=} \\
=\left[\frac{\partial X\left(-t_{1},(x)_{s}\right)}{\partial(x)_{s}}\right]^{2}\left[\frac{\partial X\left(-t_{2}, X\left(-t_{1},(x)_{s}\right)\right)}{\partial X\left(-t_{1},(x)_{s}\right)}\right]^{2} S_{s}\left(-t_{1}-t_{2},(x)_{s}\right)= \\
=\left[\frac{\partial X\left(-t_{1}-t_{2},(x)_{s}\right)}{\partial(x)_{s}}\right]^{2} S_{s}\left(-t_{1}-t_{2},(x)_{s}\right) .
\end{gathered}
$$

We used that

$$
S_{s}\left(-t,(x)_{s}\right)\left[f_{s}\left((x)_{s}\right) g_{s}\left((x)_{s}\right)\right]=\left[S_{s}\left(-t,(x)_{s}\right) f_{s}\left((x)_{s}\right)\right]\left[S_{s}\left(-t,(x)_{s}\right) g_{s}\left((x)_{s}\right)\right] .
$$

These equalities are equivalent to the following ones: 


$$
J\left(-t_{1}\right) S\left(-t_{1}\right) J\left(-t_{2}\right) S\left(-t_{2}\right)=J\left(-t_{1}-t_{2}\right) S\left(-t_{1}-t_{2}\right) .
$$

One can prove likewise that

$$
J\left(-t_{2}\right) S\left(-t_{2}\right) J\left(-t_{1}\right) S\left(-t_{1}\right)=J\left(-t_{1}-t_{2}\right) S\left(-t_{1}-t_{2}\right) .
$$

Thus equality (1.15) is proved.

Using (1.13) - (1.15) we can prove the group property of the operator $U(t)$

$$
U\left(t_{1}+t_{2}\right)=U\left(t_{1}\right) U\left(t_{2}\right)=U\left(t_{2}\right) U\left(t_{1}\right) .
$$

Indeed

$$
\begin{gathered}
U\left(t_{1}+t_{2}\right)=e^{\int d x} J\left(-t_{1}-t_{2}\right) S\left(-t_{1}-t_{2}\right) e^{-\int d x}= \\
=e^{\int d x} J\left(-t_{1}\right) S\left(-t_{1}\right) J\left(-t_{2}\right) S\left(-t_{2}\right) e^{-\int d x}= \\
=e^{\int d x} J\left(-t_{1}\right) S\left(-t_{1}\right) e^{-\int d x} e^{\int d x} J\left(-t_{2}\right) S\left(-t_{2}\right) e^{-\int d x}=U\left(t_{1}\right) U\left(t_{2}\right)
\end{gathered}
$$

because $e^{-\int d x} e^{\int d x}=I$.

By similar calculation one obtains

$$
U\left(t_{1}+t_{2}\right)=U\left(t_{2}\right) U\left(t_{1}\right)
$$

and (1.16) is proved.

1.4. Strong continuity of the group $U(t)$. We have proved that evolution operators $U(t), t \geq 0$, are bounded in $L_{1}$, according to (1.12), and possess the group property according to (1.16).

Now we prove that evolution operator $U(t)$ is strongly continuous, i.e., that

$$
\lim _{\Delta t \rightarrow 0}\|U(t+\Delta t) f-U(t) f\|=0, \quad f \in L_{1} .
$$

We will follow books $[2,3]$ with some modification.

From boundness of $U(t)$ and its group property it is sufficient to prove that

$$
\lim _{\Delta t \rightarrow 0}\|U(\Delta t) f-f\|=0 .
$$

The operator $U(\Delta t)$ is product of the operators $e^{\int d x} J(-\Delta t) S(-\Delta t) e^{-\int d x}$, where operators $e^{ \pm \int d x}$ are bounded in $L_{1}$. It means that in order to prove (1.18) it is sufficient to prove strong continuity of group $J(-t) S(-t)$ for $f \in L_{1}^{0}$. It follows from the fact that

$$
e^{-\int d x} J(-\Delta t) S(-\Delta t) e^{-\int d x} f-f=e^{\int d x}\left[J(-\Delta t) S(-\Delta t) e^{-\int d x} f-e^{-\int d x} f\right]
$$

and that $e^{-\int d x} f \in L_{1}^{0}$ when $f \in L_{1}^{0}$. Recall that subspace $L_{1}^{0}$ consists of finite sequences of functions $f_{s}\left((x)_{s}\right) \in L_{s}^{0}$. Functions $f_{s}\left((x)_{s}\right) \in L_{s}^{0}$ are continuously differentiable with compact support and equal to zero in some neighborhood of the forbidden configuration. Subspace $L_{s}^{0}$ is everywhere dense in $L_{s}$ and $L_{1}^{0}$ in $L_{1}$ [1].

Note that strong continuity of $J(-t) S(-t)$ has already been proved in [1] (see (2.1)(2.3)). Indeed, it has been proved that functions $[J(-t) S(-t) f]_{s}\left((x)_{s}\right), f_{s}\left((x)_{s}\right) \in L_{s}^{0}$ are continuous functions with respect to $t, t \geq 0$, uniformly with respect to $(x)_{s}$ on compacts. Therefore

$$
\lim _{\Delta t \rightarrow 0} \int\left|J(-\Delta t) S(-\Delta t) f_{s}\left((x)_{s}\right)-f_{s}\left((x)_{s}\right)\right| d(x)_{s}=0
$$

ISSN 1027-3190. Укр. мат. журн., 2006, 58, № 3 
because integrand has compact support and tends to zero as $\Delta t \rightarrow 0$ uniformly on compacts with respect to $(x)_{s}$.

Taking into account that $f \in L_{1}^{0}$ is finite sequence, we proved that

$$
\lim _{\Delta t \rightarrow 0}\|U(\Delta t) f-f\|=0, \quad f \in L_{1}^{0} .
$$

It follows from boundness of $U(t)$ and taking into account that $L_{1}^{0}$ is everywhere dense in $L_{1}$ that

$$
\lim _{\Delta t \rightarrow 0}\|U(\Delta t) f-f\|=0
$$

for arbitrary $f \in L_{1}$. Thus strong continuity of the evolution operator $U(t)$ (1.17) is proved.

We summarize obtained above results in the following theorem.

Theorem I. The evolution operators $U(t), t \geq 0$, are a group of bounded strongly continuous operators in $L_{1}$.

2. Infinitesimal generator of group $U(t)$ and solution of the BBGKY hierarchy in $L_{1}$. 2.1. Infinitesimal operator of group $U(t)$. As known, the group of bounded strongly continuous operators $U(t)$ in $L_{1}$ is strongly differentiable and its infinitesimal generator is defined on everywhere dense set in $L_{1}$. Now we proceed to determine this infinitesimal generator. We will follow the books [2, 3] with some modification.

Theorem II. The infinitesimal generator $B$ of the group $U(t)$ is closed; its spectrum is concentrated on the imaginary axis. On the set $L_{1}^{0}$, everywhere dense in $L_{1}, B$ coincides with the operator $B=-\mathcal{H}+\left[\int d x, \mathcal{H}\right]$, or, componentwise,

$$
\begin{gathered}
(B f)_{s}\left(x_{1}, \ldots, x_{s}\right)= \\
=-\mathcal{H} f_{s}\left(x_{1}, \ldots, x_{s}\right)+a^{2} \sum_{i=1}^{s} \int d p_{s+1} \int_{S_{+}^{2}} d \eta\left\langle\eta,\left(p_{i}-p_{s+1}\right)\right\rangle \times \\
\times\left[\frac{1}{(1-2 \varepsilon)^{2}} f_{s+1}\left(x_{1}, \ldots, q_{i}, p_{i}^{*}, \ldots, x_{s}, q_{i}-a \eta, p_{s+1}^{*}\right)-\right. \\
\left.-f_{s+1}\left(x_{1}, \ldots, q_{i}, p_{i}, \ldots, x_{s}, q_{i}+a \eta, p_{s+1}\right)\right] \\
\mathcal{H} f_{s}\left(x_{1}, \ldots, x_{s}\right)=-\sum_{i=1}^{s} p_{i} \frac{\partial}{\partial q_{i}} f_{s}\left(x_{1}, \ldots, x_{s}\right),
\end{gathered}
$$

with the following boundary condition:

If $q_{i}-q_{j}=a \eta,\left\langle\eta,\left(p_{i}-p_{s+1}\right)\right\rangle>0$, then momenta $p_{i}, p_{j}$ should be replaced by $p_{i}^{*}, p_{j}^{*}$ and $f_{s+2}\left(x_{1}, \ldots, x_{s}, q_{s+1}, p_{s+1}, q_{s+1}-a \eta, p_{s+2}\right)$ by $\frac{1}{(1-2 \varepsilon)^{2}} f_{s+2}\left(x_{1}, \ldots, x_{s}, q_{s+1}\right.$, $\left.p_{s+1}^{*}, q_{s+1}-a \eta, p_{s+2}^{*}\right)$. The operator $\mathcal{H}$ with this boundary condition is the infinitesimal generator of the group $J(-t) S(-t)$.

On set $L_{1}^{0}$ the operators $B$ and $U(t)$ commute,

$$
B U(t)=U(t) B
$$


and

$$
\frac{d}{d t} U(t)=B U(t)=U(t) B
$$

Proof. The proof of Theorem II coincides completely with the corresponding proof for a system of hard spheres with elastic collisions (see [2, 3]). As for the systems of hard spheres with elastic collisions the crucial point is identity

$$
\left[\int d x,\left[\int d x, \mathcal{H}\right]\right] f=0, \quad f \in L_{1}^{0} .
$$

In our case of systems of hard spheres with inelastic collisions, projection of this identity on $s$-particle subspace has the following form:

$$
\begin{gathered}
\int d x_{s+2} a^{2} \int d p_{s+1} \int_{S_{+}^{2}} d \eta\left\langle\eta,\left(p_{s+1}-p_{s+2}\right)\right\rangle \times \\
\times\left[\frac{1}{(1-2 \varepsilon)^{2}} f_{s+2}\left(x_{1}, \ldots, x_{s}, q_{s+1}, p_{s+1}^{*}, q_{s+1}-a \eta, p_{s+2}^{*}\right)-\right. \\
-f_{s+2}\left(x_{1}, \ldots, x_{s}, q_{s+1}, p_{s+1}, q_{s+1}+a \eta, p_{s+2}\right]
\end{gathered}
$$

and it is equal to zero, as follows from [1] (formulae (3.5)).

Note that functions $\left(J(-t) S(-t) f_{s+1}\right)\left(x_{1}, \ldots, x_{s+1}\right)$ are (possibly) different from zero in a neighborhood of forbidden configurations, where the functions $f_{s+1}\left(x_{1}, \ldots\right.$ $\left.\ldots, x_{s+1}\right) \in L_{s+1}^{0}$ vanish. From this it follows that the functions $(U(t) f)_{s+1}\left(x_{1}, \ldots\right.$ $\left.\ldots, x_{s+1}\right), f \in L_{1}^{0}$ are (possibly) different from zero in a neighborhood of forbidden configurations and on the hypersurfaces $\left|q_{i}-q_{s+1}\right|=a, i=1, \ldots, s$. This means that the second term in formula (see (2.1))

$$
\left(\frac{d}{d t} U(t) f\right)_{s}\left(x_{1}, \ldots, x_{s}\right)=(B U(t) f)_{s}\left(x_{1}, \ldots, x_{s}\right)
$$

is different from zero.

2.2. Existence of solutions of the BBGKY hierarchy. The BBGKY hierarchy (1.2) is the evolution equation for the infinite sequence $\rho(t)$ of correlation functions $\rho(t)=$ $=\left(\rho_{1}\left(t, x_{1}\right), \ldots, \rho_{s}\left(t, x_{1}, \ldots, x_{s}\right), \ldots\right)$. This equation reads

$$
\frac{d \rho(t)}{d t}=B \rho(t)=-\mathcal{H} \rho(t)+\left[\mathcal{H}, \int d x\right] \rho(t)
$$

with initial condition

$$
\left.\rho(t)\right|_{t=0}=\rho(0) .
$$

The operator B is the infinitesimal generator of the group $U(t)$ (1.7).

One can consider the BBGKY hierarchy as the abstract evolution equation (2.2) in the Banach space $L_{1}$ with initial data $(2.3), \rho(0) \in L_{1}$. Then in follows from Theorem II that

$$
\rho(t)=U(t) \rho(0) .
$$

is the strong solution of the Cauchy problem for the BBGKY hierarchy (2.2) with initial data $\rho(0) \in L_{1}^{0}$. For general initial data $\rho(0) \in L_{1}^{0}, \rho(t)=U(t) \rho(0)$ is a generalized solution in the following sense. The strong solutions exist for $\rho(0) \in L_{1}^{0}$ and are represented 
by formulae (2.4). The set $L_{1}^{0}$ is everywhere dense in $L_{1}$ and for arbitrary $\rho(0) \in L_{1}^{0}$ there exists a sequence $\rho(0)^{i} \in L_{1}^{0}$ which strongly converges to $\rho(0)$. From the boundedness of $U(t)$ it follows that the sequence $U(t) \rho(0)^{i}$ also converges to $U(t) \rho(0)$ and in this sense $\rho(t)=U(t) \rho(0)$ is a generalized solution of the BBGKY hierarchy.

The above results can be summarized in the following theorem.

Theorem III. The Cauchy problem for the BBGKY hierarchy (2.2) has a solution in $L_{1}$, given by formula (2.4). For initial data $\rho(0) \in L_{1}^{0} \subset L_{1}$ this solution is strong, for arbitrary initial data $\rho(0) \in L_{1}$ it is a generalized solution.

2.3. States of infinite systems. Solutions of the hierarchy (1.2) or (2.2) constructed above describe states of finite systems, because the average number $\bar{N}$ of particles corresponding to the state $\rho(t) \in L_{1}, \rho(0) \in L_{1}$,

$$
\bar{N}=\int \rho_{1}\left(t, x_{1}\right) d x_{1}<\infty
$$

is finite. This means that solutions of the hierarchy (1.2) or (2.2) for initial data $\rho(0) \in$ $\in L_{1}$ can not describe states of infinite systems, i.e., system consisting of infinite average number of particles situated in the entire phase space on admissible configurations.

Usually, in the case of elastic collisions, perturbations of equilibrium states of infinite systems - i.e., Gibbs states for given temperature and density are considered, as initial data for an infinite system. We hope to realize this approach in the case of an infinite system of hard spheres with inelastic collisions.

As it has been pointed in paper [1] hierarchy (1.2) or (2.2), has the stationary solution

$$
\rho_{s}\left((x)_{s}\right)=\prod_{i=1}^{s} \delta\left(p_{i}-p\right) \prod_{i<j=1}^{s} \Theta\left(\left|q_{i}-q_{j}\right|-a\right), \quad s \geq 1,
$$

where $p$ is arbitrary momentum. It would be naturally to consider as initial data for infinite system some perturbation of sequence (2.6). For example

$$
\rho_{s}\left((x)_{s}\right)=\prod_{i=1}^{s} \frac{1}{(2 \pi \beta)^{\frac{3}{2}}} e^{-\beta\left(p_{i}-p\right)^{2}} \prod_{i<j=1}^{s} \Theta\left(\left|q_{i}-q_{j}\right|-a\right), \quad s \geq 1,
$$

where $\beta$ is some positive parameter, $\beta>0$. Sequence (2.7) can be considered as a perturbation of sequence (2.6), because it converges as $\beta \rightarrow 0$ to it, in the sense of generalized functions.

1. Caraffini G. L., Petrina D. Ya. Analogue of Liouville equation and BBGKY hierarchy for a system of hard spheres with inelastic collisions // Ukr. Math. J. - 2005. - 57, № 6. - P. 818 - 839.

2. Cercignani C., Gerasimenko V. I., Petrina D. Ya. Many-particle dynamics and kinetic equations. - Dordrecht: Kluwer Acad./Plenum Publ., 1997. - 252 p.

3. Petrina D. Ya., Gerasimenko V. I., Malyshev P. V. Mathematical foundations of classical statistical mechanics. Continuous systems. - Second ed. - London; New York: Taylor and Francis Sci. Publ., 2002. $352 \mathrm{p}$.

Received 08.02.2005 